\title{
Attenuated Psychosis Syndrome in DSM-5
}

\author{
Ming Tsuang a,b,c, Jim Van Os ${ }^{\mathrm{d}, \mathrm{e}}$, Rajiv Tandon ${ }^{f}$, Deanna M. Barch ${ }^{\mathrm{g}}$, Juan Bustilloh, \\ Wolfgang Gaebel', Raquel E. Gurj, Stephan Heckers ${ }^{k}$, Dolores Malaspinal,m, Michael J. \\ Owen $^{\mathrm{n}}$, Susan Schultz ${ }^{\circ}$, and William Carpenter ${ }^{\mathrm{P}}$
}

aCenter for Behavioral Genomics, Department of Psychiatry and Institute of Genomic Medicine, University of California, San Diego, CA, USA b Veterans Affairs San Diego Healthcare System, San Diego, CA, USA CHarvard Institute of Psychiatric Epidemiology and Genetics, Harvard School of Public Health, Boston, Massachusetts, USA dMaastricht University Medical Centre, South Limburg Mental Health Research and Teaching Network, EURON, Maastricht, The Netherlands 'King's College London, King's Health Partners, Department of Psychosis Studies, Institute of Psychiatry, London, United Kingdom fDepartment of Psychiatry, University of Florida Medical School, Gainesville, FL, USA gDepartments of Psychology, Psychiatry and Radiology, Washington University, St. Louis, MO, USA hDepartment of Psychiatry, University of New Mexico, Albuquerque, NM, USA 'Department of Psychiatry, University of Dusseldorf, Germany kDepartment of Psychiatry, Vanderbilt University, Nashville, TN, USA 'Department of Psychiatry, New York University, New York, NY iDepartments of Psychiatry, Neurology and Radiology, Perlman School of Medicine, University of Pennsylvania, Philadelphia, PA, USA ${ }^{\mathrm{m}}$ Creedmoor Psychiatric Center, New York State Office of Mental Health, USA nMRC Centre for Neuropsychiatric Genetics and Genomics and Neuroscience and Mental Health Research Institute, Cardiff University, Cardiff, Wales ${ }^{\circ}$ Department of Psychiatry, University of lowa School of Medicine, lowa City, IA, USA PDepartment of Psychiatry, Maryland Psychiatric Research Center, Baltimore, MD, USA

\section{Abstract}

Despite advances in the treatment of schizophrenia over the past half-century, the illness is frequently associated with a poor outcome. This is principally related to the late identification and intervention in the course of the illness by which time patients have experienced a substantial amount of socio-occupational decline that can be difficult to reverse. The emphasis has therefore shifted to defining psychosis-risk syndromes and evaluating treatments that can prevent transition to psychosis in these ultra-high risk groups. To consider the appropriateness of adding psychosis risk syndrome to our diagnostic nomenclature, the Psychotic Disorders Workgroup extensively reviewed all available data, consulted a range of experts, and carefully considered the variety of expert and public comments on the topic. It was clear that reliable methods were available to define a syndrome characterized by sub-threshold psychotic symptoms (in severity or duration) and which was associated with a very significant increase in the risk of development of a fullfledged psychotic disorder (schizophrenia spectrum, psychotic mood disorder, other psychotic disorder) within the next year. At the same time, the majority of individuals with "attenuated psychotic symptoms" had one or more other current psychiatric comorbid conditions (usually

\footnotetext{
C 2013 Elsevier B.V. All rights reserved.

Corresponding Author: Ming T. Tsuang, M.D., Ph.D., D.Sc., Department of Psychiatry, University of California, San Diego, MC 0603, 9500 Gilman Drive, La Jolla, CA 92093, Phone: (858) 822-2464 Fax: (858) 822-2469, mtsuang@ucsd.edu.

Publisher's Disclaimer: This is a PDF file of an unedited manuscript that has been accepted for publication. As a service to our customers we are providing this early version of the manuscript. The manuscript will undergo copyediting, typesetting, and review of the resulting proof before it is published in its final citable form. Please note that during the production process errors may be discovered which could affect the content, and all legal disclaimers that apply to the journal pertain.
} 
mood or anxiety disorders, substance use disorder; Fusar-Poli 2012) and exhibited a range of psychiatric outcomes other than conversion to psychosis (significant proportions either fully recover or develop some other psychiatric disorder with a minority developing a psychotic disorder). Whereas the reliability of the diagnosis is well established in academic and research settings, it was found to be less so in community and other clinical settings. Furthermore, the nosological relationship of Attenuated Psychosis Syndrome (APS) to schizotypal personality disorder and other psychiatric conditions was unclear. Further study will hopefully resolve these questions. The Workgroup decided to recommend the inclusion of Attenuated Psychosis Syndrome as a category in the appendix (Section 3) of DSM-5 as a condition for further study.

\section{Introduction}

Despite therapeutic advances over the past half-century, schizophrenia continues to be a debilitating disorder with profound lifelong impairments in social and vocational functioning for most of those with the condition. Much of the decline occurs early in the course of the illness and overall outcome is directly correlated with functional ability prior to onset of psychosis and inversely correlated with duration of untreated psychosis (Carpenter, 2009; Woods et al., 2010; Boonstra et al., 2012). These facts have provided the impetus to early intervention efforts. Reducing treatment delay from the onset of the initial psychotic episode by early diagnosis and effective treatment has yielded only modest improvements in outcome for individuals with schizophrenia, however, leading to interest in possibilities for intervention even earlier in the course of the illness (i.e., before onset of psychosis; Fusar-Poli et al., 2013). This knowledge has lead to interest from several centers, including the North American Prodromal Longitudinal Study (NAPLS, Addington et al., 2007), in developing early psychosis detection, intervention, and treatment programs.

In order to address this need and in light of improvements in outcome observed in various early psychosis intervention programs, the Psychotic Disorders workgroup considered the addition in DSM-5 of a new category of "Psychosis Risk Syndrome" or "Attenuated Psychosis Syndrome" to describe a condition with recent onset of modest, psychotic-like symptoms and clinically relevant distress and disability. These patients also are at significantly increased risk of conversion to a full-blown psychotic disorder (Fusar-Poli et al., 2012). Based on a review of the data relatively early in the process, it was realized that it may be premature to recommend a new category primarily based on future risk (i.e., "Psychosis Risk Syndrome") and not on current clinical need (Carpenter and van Os, 2011; Tandon, 2012). Data revealed that a majority of individuals with this condition did not go on to develop a psychotic disorder and that most individuals with this condition had additional relevant clinical needs other than the risk of conversion to psychosis. Consequently, a condition that described current clinical need - Attenuated Psychosis Syndrome (APS) - was considered instead. In contrast to "Psychosis Risk Syndrome", APS describes a currently relevant clinical condition leading to help-seeking, with many more clinical outcomes other than conversion to psychosis. The main considerations with respect to APS involved matters of reliability of diagnosis in routine clinical settings and whether it had more validity and provided greater clinical utility than current classification systems (Carpenter and van Os, 2011; Tandon and Carpenter, 2012). The relationship of APS to related diagnostic categories such as schizotypal personality disorder was also evaluated. In addition to reviewing all available data, the Psychotic Disorders workgroup consulted a range of experts, and considered a variety of public and expert comments on the topic.

\section{Proposed Clinical Criteria for APS}

A. At least one of the following symptoms are present in attenuated form with sufficient severity and/or frequency to warrant clinical attention: 
1. delusions/delusional ideas

2. hallucinations/perceptional abnormalities

3. disorganized speech/communication

B. Symptoms in Criterion A must be present at least once per week for the past month.

C. Symptoms in Criterion A must have begun or worsened in the past year.

D. Symptoms in Criterion A are sufficiently distressing and disabling to the individual and/or legal guardian to lead them to seek help.

E. Symptoms in Criterion A are not better explained by any other DSM-5 diagnosis, including Substance-Related Disorders.

F. Clinical criteria for a Psychotic Disorder have never been met (McGlashan et al. 2010).

\section{Rationale}

\section{A. Does the new diagnosis address a current unmet clinical need?}

A vast majority of individuals who go on to develop schizophrenia or other psychotic disorder exhibit a range of psychiatric symptoms in the period prior to their initial psychotic episode. During this period, many such individuals experience decline in their academicoccupational and other aspects of social functioning that are difficult to reverse when they seek treatment after onset of the psychotic disorder (Tandon and Maj, 2008). Currently, there is no diagnostic category to define individuals who are experiencing such psychopathology and are at significantly higher risk for developing schizophrenia or other psychotic disorder. This proposed disorder category is intended for use when there is no existing diagnostic category to better define individuals who are experiencing such psychopathology and are at significantly higher risk for developing schizophrenia or other psychotic disorder. For example, the recent onset and transitory criteria preclude a diagnosis of schizotypal personality diagnosis and the sub threshold manifestations of psychosis-like symptoms do not meet criteria for a full psychotic disorder. The current lack of an appropriate diagnosis in DSM-5 prevents such individuals from obtaining appropriate clinical attention that might provide current relief and possibly prevent future adverse psychiatric outcomes. Several groups around the world have devised diagnostic criteria and assessment tools (Miller et al., 2002 and 2003; Yung et al., 2005) to reliably identify such "ultra-high risk" individuals who have a significantly greater likelihood than the general population of developing a psychotic disorder over the next two and a half years (Cannon et al., 2008). In general, it appears that about $1 / 3$ of Ultra High Risk (UHR) cases convert to psychosis (Fusar-Poli et al., 2012a; Gee \& Cannon 2012). Although a range of interventions (including careful observation and monitoring) appear to be effective in reducing rates of conversion to psychosis, they are as yet inadequately differentiated. Close follow-up is important and should include assessment for conversion to psychosis as also assessment for development or persistence of other psychiatric conditions and provision of appropriate treatment.

\section{B. Prevalence in Epidemiological Samples}

Relatively little is known about the prevalence of individuals with attenuated psychotic symptoms in the general population. Meta-analyses suggest that the prevalence of individuals with attenuated psychotic symptoms (which is not the same as APS where help seeking behaviors are sought) in the general population is around 5 percent (Linscott and van Os, 2012); only a small proportion of these seek help with mental health services and 
would be eligible for a diagnosis of APS, which is defined in terms of help-seeking and clinically relevant distress and dysfunction.

\section{Information about Reliability of Proposed Criteria}

In research settings, the reliability of the proposed criteria is moderate before training (Cohen's kappa ranging from 0.3-0.5) and high after training (Cohen's kappa ranging from 0.75-0.90).

Reliability data in general clinical settings are limited. The DSM-5 field trial provided too small a sample for an informative test of reliability (Regier et al., 2013).

\section{Data on Validity}

\section{Antecedent Validity}

Limited information is available in the published literature.

\section{Concurrent validity}

1. Cognition-Two meta-analyses by Giuliano et al. (2012) and Fusar-Poli et al. (2012b) have been published. The latter (19 studies, 1188 High Risk [HR] and 1029 Controls) showed HR subjects were impaired on tests of general intelligence, executive functions, verbal/visual memory, verbal fluency, attention and working memory and social cognition. Transition to psychosis was associated with deficits in the verbal fluency and memory domains.

\section{Imaging}

a) Neuroanatomy: Recent meta-analysis of Voxel Based Morphometry (VBM) studies. (Pantelis et al., 2003; Fusar-Poli et al., 2012c) are consistent with findings of the largest multicenter VBM structural study by Mechelli et al. (2011) in $182 \mathrm{HR}$ and 167 controls showing that the HR group as a whole had less gray matter volume than did controls in the frontal regions. The HR who later developed psychosis had less gray matter volume in the parahippocampal cortex than the HR subgroup who did not convert.

b) Neurochemistry: Howes et al. $(2009,2011)$ showed an association between elevation of pre-synaptic dopamine synthesis capacity and transition to psychosis. Multimodal fMRIPET and fMRI-MRS data also provide support for the validity of a HR diagnosis (Fusar-Poli et al., 2010, 2011).

\section{Predictive validity}

\section{Outcomes}

Transition risks: A meta analysis in 27 High Risk \{HR (At Risk Mental States, APS) \} studies (Fusar-Poli et al., 2012a), relating to 2502 HR subjects showed the following rates of conversion to psychosis:

$18 \%$ (95\%CI 12.3\%-24.9\%) after six months of follow-up,

$22 \%$ (95\%CI 16.6\%-27.8\%) after one year,

$29 \%$ \{95\%Confidence Interval(CI) $23.3 \%-35.7 \%\}$ after two years, and

$36 \%(29.6 \%-42.5 \%)$ after three years.

These risks of developing a psychotic disorder summed across available studies are substantially greater than in the general population- $22 \%$ at one year follow-up compared to 
a $0.015 \%$ annual incidence of schizophrenia (Fusar-Poli et al., 2012a; Tandon et al, 2008). The risk is moderated by increasing age of HR subjects, a modest but significant effect towards declining transition risks in the most recent published papers and the effects of treatment (Yung et al., 2007).

Diagnostic outcomes: In a meta-analysis of studies in which specific diagnostic outcomes were noted for HR individuals who transitioned to psychosis $\{\mathrm{n}=560$ High Risk for Psychosis (HRP) with transition to psychosis \}: $73 \%$ converted to schizophrenia spectrum disorders (schizophrenia, schizophreniform, schizoaffective) and 11\% psychotic mood disorders affective (psychotic depression, bipolar psychosis) (Relative Risk $=5.4$ ) (FusarPoli et al., 2012d).

Remission: A systematic review (Simon et al., 2011) uncovering six studies reported remission rates from initial HR status (proportion of remissions ranged from $15.4 \%$ to $54.3 \%)$.

2. Interventions-Controlled clinical trials testing efficacy of various interventions are sparse and of small sample size, with control groups frequently receiving treatment that may be effective. A recent review of seven studies with several different therapeutic approaches suggested that the experimental treatment was superior to usual treatment in prevention of progression to full psychosis, with a transition rate average across studies of $7.6 \%$ for experimental treatment and 23\% for usual treatment (Fusar-Poli et al, 2013). The most provocative individual study was a random assignment placebo controlled study in which 12 weeks of omega-3 fatty acids was robustly superior to placebo in preventing psychosis over the next 40 weeks, though this single study requires replication (Amminger et al., 2010). There are mixed results with regard to the effectiveness of CBT in preventing transition of the HR state to psychosis (Morrison et al., 2012; van der Gaag et al, 2012).

\section{Recommendations of Workgroup}

Based on the evidence above, several experts advocated inclusion of APS in the main body of the DSM-5 diagnostic manual (Woods et al., 2010), whereas others suggested that it should be broadened to a general early syndrome of significant psychopathology in line with the staging model of psychopathology (McGorry and van Os, 2013). There was a uniform consensus among the experts that Attenuated Psychosis Syndrome is a condition that warrants systematic attention (Yung et al., 2012), although experts disagreed as to whether this diagnosis should be placed in the main body of the diagnostic manual or whether it should be placed in the appendix (Section 3) as a condition for further systematic study (Corcoran et al., 2010; Drake and Lewis, 2010; Woods et al., 2010; Carpenter and van Os, 2011; Tandon et al., 2012). The Work Group believed that one fundamental question was whether APS would be reliably diagnosed by non-experts in ordinary clinical settings. Without field trial data supporting reliability (Regier et al., 2013), it was clear that APS would not be considered further for the main text (Tandon and Carpenter, 2013). If the field trials had been adequate and supportive of reliability, an interesting debate would have commenced as to whether to recommend that APS be included in the main text, Section III, or to make no recommendation for inclusion. Five principal areas of debates put forth by the experts who recommend inclusion of APS in the appendix instead of the main body of the diagnostic manual were:

- A majority of individuals with current APS have some other current psychiatric comorbidity (frequently depressive, anxiety, or substance use disorder, Fusar-Poli et al., 2012) which warrants appropriate treatment at the current time. The alternative view is that these symptoms are common in many disorders including 
psychotic disorders, merit clinical attention, but are not established as the basic disorder in research to date relevant to APS.

- A substantial proportion of individuals with APS do not go on to develop major psychopathology. Whereas conversion to schizophrenia or other full-blown psychosis is one possible outcome of APS, this occurs in a minority of persons. The alternative view is that many of the patients continue to have symptoms and functional impairments that merit attention (Addington et al., 2011), and that the value of identifying APS is not only determined by subsequent transition to psychosis, although secondary prevention of full psychosis is a desirable effect as well.

- It is unclear if APS represents a trait or state vulnerability (for increased risk of development of a psychotic disorder) and its relationship to schizotypal personality disorder is not clear. This is countered by noting that the criteria for APS focus on state phenomena, and that individual for whom APS is an appropriate diagnosis do not meet trait requirements for a diagnosis of schizotypal personality disorder. Further, many individuals with schizotypal personality disorder do not progress to schizophrenia or a related full psychosis, though rates of progression may be higher in young persons with schizotypal personality disorder.

- It is unclear if the distress and/or disability resulting in help-seeking behavior by this group of individuals is related to APS or the "comorbid" mental disorder; helpseeking is part of its definition in DSM-5. The counter-argument is that the APS criteria require that distress, dysfunction and/or impairment be related to the symptom criteria. This does not exclude anxiety or depression from contributing to help-seeking, but these would be judged to be associated or secondary features, not a disorder that accounts for the full clinical presentation. If anxiety, for example, is considered co-morbid, the question remains as to co-morbid with what? APS would be the proposed answer.

- There was concern about potential stigma and inappropriate antipsychotic utilization in individuals with APS (Jacobs et al., 2012). The counter-argument was that a new APS category will educate clinicians about the relative lack of utility of antipsychotic medications in this population (Stafford et al., 2013) and may actually reduce inappropriate antipsychotic use among youth. Furthermore, any stigma is principally related to behaviors associated with a diagnosis of APS rather than the diagnosis itself, thus an APS category might lead to a reduction in traumatic experiences (Addington et al., 2013).

Despite the validating evidence in research to date (Fusar-Poli et al, 2013), the failed reliability field trials precluded further consideration for inclusion as a new disorder in the main text. Studies to date have been organized around the concept of the schizophrenia prodrome and results support APS as currently defined to be part of a schizophrenia spectrum disorder. It is expected that future studies that include risk or prodromal features of other disorders associated with psychosis may broaden the definition and change the proportion of transition cases that belong in the schizophrenia spectrum.

The Workgroup determined that more work was necessary before APS could be considered for inclusion in the main body of DSM (Yung et al., 2012). From a clinical point of view, immediate needs include:

i. knowledge on how APS works in ordinary clinical settings in terms of reliability and predictive utility; 
ii. at what stage in the development of APS related pathology is it optimal to define a disorder; and

iii. whether a disorder, so defined, enhances the acquisition of therapeutic knowledge.

The workgroup concluded that there were strong reasons to continue to evaluate this clinical entity and provision of specific criteria and description would help in this effort.

Furthermore, it was also recognized that early detection and intervention is a high value throughout medicine, and that secondary prevention of full psychosis may offer substantial life course benefits. It seems likely that psychiatry will move in this direction with a number of disorders in the future. For reasons reviewed above, APS is being assigned to Section III for further study.

\section{Acknowledgments}

Without the efforts of Thomas H. McGlashan, Barbara C. Walsh, and Scott W. Woods of Yale University's Department of Psychiatry, the completion of this work would not have been possible.

\section{References}

Addington J, Cadenhead KS, Cannon TD, et al. North American Prodrome Longitudinal Study: a collaborative multisite approach to prodromal schizophrenia research. Schizophr Bull. 2007; 33:665-672. [PubMed: 17255119]

Addington J, Cornblatt BA, Cadenhead KS, et al. At clinical high risk for psychosis: outcome for nonconvertors. Am J Psychiatry. 2011; 168:800-805. [PubMed: 21498462]

Addington J, Stowkowy J, Cadenhead KS, Cornblatt BA, McGlashan TH, Perkins DO, Seidman LJ, Tsuang MT, Walker EF, Woods SW, Cannon TD. Early traumatic experiences in those at clinical high risk for psychosis. Early Interv Psychiatry. 2013 Jan 24. [Epub ahead of print].

Amminger GP, Schafer MR, Papageorgiou K, et al. Long-chain omega-3 fatty acids for indicated prevention of psychotic disorders: a randomized, placebo-controlled trial. Arch Gen Psychiatry. 2010; 67:146-154. [PubMed: 20124114]

Boonstra N, Klaasen R, Sytema S, et al. Duration of untreated psychosis and negative symptoms- A systematic review and meta-analysis of individual patient data. Schizophr Res. 2012; 142:12-19. [PubMed: 23025994]

Cannon TD, Cadenhead K, Cornblatt B, et al. Prediction of psychosis in youth at high clinical risk: a multisite longitudinal study in North America. Arch Gen Psychiatry;. 2008; 65:28-37.

Carpenter WT. Anticipating DSM-V. Should psychosis risk become a diagnostic class? Schizophr Bull. 2009; 35:841-845. [PubMed: 19633215]

Carpenter WT, van Os J. Should attenuated psychosis syndrome be a DSM-5 diagnosis? Am J Psychiatry. 2011; 168:460-463. [PubMed: 21536700]

Corcoran CM, First MB, Cornblatt B. The psychosis-risk syndrome and its proposed inclusion in the DSM-V. A risk-benefit analysis. Schizophr Res. 2010; 120:16-22. [PubMed: 20381319]

Drake RJ, Lewis SW. Valuing prodromal psychosis: what do we get and what is the price? Schizophr Res. 2010; 120:38-41. [PubMed: 20417060]

Fusar-Poli P, Howes OD, Allen P, et al. Abnormal frontostriatal interactions in people with prodromal signs of psychosis: a multimodal imaging study. Arch Gen Psychiatry. 2010; 67:683-691. [PubMed: 20603449]

Fusar-Poli P, Stone J, Broome M, et al. Thalamic glutamate levels predicts cortical response during executive functioning in subjects at high risk for psychosis. Arch Gen Psychiatry. 2011; 68:881890. [PubMed: 21536967]

Fusar-Poli P, Bonoldi I, Yung AR, et al. Predicting psychosis: a meta-analysis of transition outcomes in individuals at high clinical risk. Arch Gen Psychiatry. 2012a; 69:1-10.

Fusar-Poli P, Deste G, Smieskova R, et al. Cognitive functioning in prodromal psychosis: a metaanalysis. Arch Gen Psychiatry. 2012b; 69:562-571. [PubMed: 22664547] 
Fusar-Poli P, Radua J, McGuire P, Borgwardt S. Neuroanatomical Maps of Psychosis Onset: Voxelwise Meta-Analysis of Antipsychotic-Naive VBM Studies. Schizophr Bull. 2012c; 38:1297-1307. [PubMed: 22080494]

Fusar-Poli P, Bechdolf A, Taylor, et al. At risk for schizophrenic or affective psychosis? A metaanalysis of ICD/DSM diagnostic outcomes in individuals at high clinical risk. Schizophr Bull Schizophr Bull. 2012d Epub ahead of print.

Fusar-Poli P, Borgwardt S, Bechdolf A, et al. The psychosis high risk state: a comprehensive state of the art review. Arch Gen Psychiatry. 2013; 70:107-120.

Fusar-Poli P, Nelson B, Valmaggia L, Yung AR, McGuire PK. Comorbid Depressive and Anxiety Disorders in 509 Individuals With an At-Risk Mental State: Impact on Psychopathology and Transition to Psychosis. Nov 22. Schizophr Bull. 2012

Gee DG, Cannon TD. Prediction of conversion to psychosis: review and future directions. Rev Bras Psiquiatr. 2011 Oct; 33 (Suppl 2):s129-42. [PubMed: 22286564]

Giuliano AJ, Li H, Mesholam-Gately RI, Sorenson SM, Woodberry KA, Seidman LJ. Neurocognition in the psychosis risk syndrome: a quantitative and qualitative review. Curr Pharm Des. 2012; 18(4):399-415. [PubMed: 22239571]

Howes O, Montgomery A, Asselin M, et al. Elevated striatal dopamine function linked to prodromal signs of schizophrenia. Archives of General Psychiatry. 2009; 66:13-20. [PubMed: 19124684]

Howes OD, Bose SK, Turkheimer F, et al. Dopamine Synthesis Capacity Before Onset of Psychosis: A Prospective [18F]-DOPA PET Imaging Study. Am J Psychiatry. 2011; 168:1311-1317. [PubMed: 21768612]

Linscott RJ, Van Os J. An updated and conservative systematic review and meta-analysis of epidemiological evidence on psychotic experiences in children and adults: on the pathway from proneness to persistence to dimensional expression across mental disorders. Psychological Medicine. 2012 Epub online publication. 10.1017/S0033291712001626

McGlashan, TH.; Walsh, BC.; Woods, SW. The Psychosis Risk Syndrome: Handbook for Diagnosis and Follow-Up. Oxford University Press; 2010.

McGorry P, Van Os J. Redeeming Diagnosis in Psychiatry: Timing versus Specificity. Lancet. 2013; 381:343-45. [PubMed: 23351805]

Mechelli A, Riecher-Rossler A, Meisenzahl EM, et al. Neuroanatomical abnormalities that predate the onset of psychosis: a multicenter study. Arch Gen Psychiatry. 2011; 68:489-495. [PubMed: 21536978]

Miller TJ, McGlashan TH, Rosen JL, et al. Prospective diagnosis of the initial prodrome for schizophrenia based on the Structured Interview for Prodromal Syndromes: preliminary evidence of interrater reliability and predictive validity. Am J Psychiatry. 2002; 159:863-5. [PubMed: 11986145]

Miller TJ, McGlashan TH, Rosen JL, et al. Prodromal assessment with the structured interview for prodromal syndromes and the scale of prodromal symptoms: predictive validity, interrater reliability, and training to reliability. Schizophr Bull. 2003; 29:703-15. [PubMed: 14989408]

Morrison AP, French P, Stewart SLK, et al. Early detection and intervention evaluation for people at risk of psychosis: multisite randomised controlled trial. BMJ. 2012; 344:e2233. [PubMed: 22491790]

Pantelis C, Velakoulis D, McGorry PD, et al. Neuroanatomical abnormalities before and after onset of psychosis: a cross-sectional and longitudinal MRI comparison. Lancet. 2003; 361:281-288. [PubMed: 12559861]

Simon AE, Velthorst E, Nieman DH, et al. Ultra high-risk state for psychosis and non-transition: A systematic review. Schizophr Res. 2011; 132:8-17. [PubMed: 21784618]

Stafford MR, Jackson H, Mayo-Wilson E, Morrison AP, Kendall T. Early interventions to prevent psychosis: systematic review and meta-analysis. BMJ. 346:f185.

Tandon N, Shah J, Keshavan MS, Tandon R. Attenuated psychosis and the schizophrenia prodrome: current status of risk identification and psychosis prevention. Neuropsychiatry. 2012; 2:345-353. [PubMed: 23125875]

Tandon R, Carpenter WT. DSM-5 status of psychotic disorders: One year pre-publication. Schizophrenia Bulletin. 2012; 39:369-370. [PubMed: 22504739] 
Tandon R, Carpenter WT. Psychotic disorders in DSM-5. Die Psychiatrie. 2013; 10:5-9.

Tandon R, Maj M. Nosological status and definition of schizophrenia: Some considerations for DSMV and ICD-11. Asian J Psychiatry. 2008; 1:22-27.

Tandon R, Keshavan MS, Nasrallah HA. Schizophrenia, "Just the Facts”. Schizophr Res. 2008; 100:420. [PubMed: 18291627]

van der Gaag M, et al. Cognitive behavioral therapy for subjects at ultrahigh risk for developing psychosis: a randomized controlled clinical trial. Schizophr Bull. 2012 Nov; 38(6):1180-8. [PubMed: 22941746]

Woods SW, Walsh BC, Saksa JR, McGlashan TH. The case for including attenuated psychotic symptom syndrome in DSM-5 as a psychosis-risk syndrome. Schizophr Res. 2010; 123:199-207. [PubMed: 20832249]

Yung AR, Yuen HP, McGorry PD, et al. Mapping the onset of psychosis: The comprehensive assessment of at-risk mental states. Aust N Z J Psychiatry. 2005; 39:964-71. [PubMed: 16343296]

Yung AR, Yuen HP, Berger G, et al. Declining transition rate in ultra high risk (prodromal) services: dilution or reduction of risk? Schizophr Bull. 2007; 33:673-681. [PubMed: 17404389]

Yung AR, Woods SW, Ruhrmann S, et al. Whither the attenuated psychosis syndrome? Schizophr Bull. 2012; 38:1130-1134. [PubMed: 23144056] 